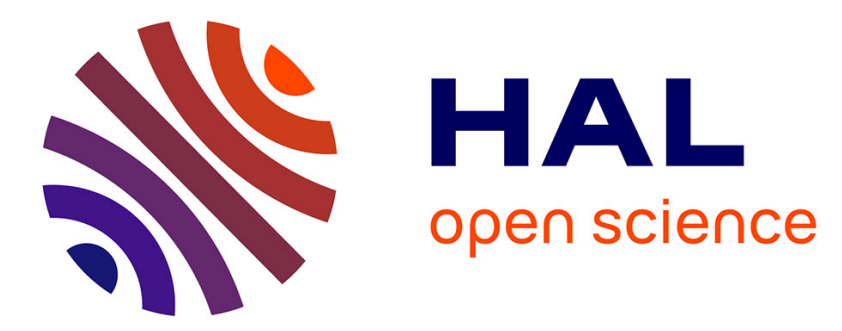

\title{
Semi-automatic abdominal aortic aneurysms geometry assessment based on 3D ultrasound
}

Laurence Rouet, Roberto Ardon, Jean-Michel Rouet, Benoît Mory, Cecile Dufour, Anne Long

\section{- To cite this version:}

Laurence Rouet, Roberto Ardon, Jean-Michel Rouet, Benoît Mory, Cecile Dufour, et al.. Semiautomatic abdominal aortic aneurysms geometry assessment based on 3D ultrasound. IEEE Ultrasonics Symposium, Oct 2010, San Diego, CA, United States. pp.201-204, 10.1109/ULTSYM.2010.5935590 . hal-00642750

\section{HAL Id: hal-00642750 \\ https://hal.science/hal-00642750}

Submitted on 18 Nov 2011

HAL is a multi-disciplinary open access archive for the deposit and dissemination of scientific research documents, whether they are published or not. The documents may come from teaching and research institutions in France or abroad, or from public or private research centers.
L'archive ouverte pluridisciplinaire HAL, est destinée au dépôt et à la diffusion de documents scientifiques de niveau recherche, publiés ou non, émanant des établissements d'enseignement et de recherche français ou étrangers, des laboratoires publics ou privés. 


\title{
Semi-automatic abdominal aortic aneurysms geometry assessment based on 3D ultrasound
}

\author{
Laurence Rouet*, Roberto Ardon*, Jean-Michel Rouet*, Benoit Mory*, Cécile Dufour* and Anne Long ${ }^{\dagger}$ \\ * MediSys Research Lab. Philips Healthcare, 92156 Suresnes, France \\ Email: laurence.rouet@philips.com \\ ${ }^{\dagger}$ Centre Hospitalier Universitaire, 51092 Reims Cedex, France
}

\begin{abstract}
This paper presents a new approach for improving the surveillance of the size of abdominal aortic aneurysms (AAA). Use of 3D ultrasound imaging combined with semi-automatic quantification provides automatic selection of the optimal plane for diameter measurement. Quantification parameters are defined to characterize the aneurysm with more accuracy. Volume imaging also provides 3D visualization of the AAA geometry and CT-like multi-planar reconstructions. Multiple volume registrations are proposed to overcome limited field of view issues. Quantification results show good correlation with 2D reference measurements and obtained Pearson correlation coefficients are significant for 30 patients.
\end{abstract}

\section{INTRODUCTION}

Abdominal aortic aneurysm (AAA) is clinically defined as a local enlargement of the aorta of more than $50 \%$ with a loss of walls parallelism. The prevalence of this disease is estimated to more than 5\% in elderly subjects [1]. The main risk associated to AAA is rupture leading to mortality in up to $80 \%$ of the cases.

The standard surveillance of AAA consists in monitoring its maximum diameter using 2D ultrasound acquisitions. The main limit of such a monitoring is a lack of measurements reproducibility. Indeed, variability of measurements between successive exams may be comparable to the amount of aneurysm growth. Variability may be due to a combination of factors: various definitions of optimal selected diameter (for example true maximum diameter or maximum antero-posterior diameters), variability of AAA geometry and orientations leading to difficult identification of optimal diameter, various protocols of acquisitions and ultrasound image quality [2], [3], [4], [5] . Another limit is that 2D ultrasound provides a fixed single view of the AAA, which may not be the optimal view and the provided measurement can not be checked offline by another observer, such as the surgeon.

An alternative to 2D ultrasound imaging is CT scanning, considered as the gold-standard for AAA diameter assessment. However, this modality is mostly used to plan surgery or to monitor endovascular repair (EVAR) follow-up. In addition radiation dose, contrast injection and cost of exams are serious issues.

Our approach consists in using 3D ultrasound acquisitions in order to overcome the above limitations. The first objective is to provide a more reproducible measurement of the maximum AAA diameter for a given patient. A second objective is to provide a more complete assessement of the AAA geometry. In particular, more precise definition of the parameters extracted and longitudinal measurement of the diameters alongside the whole AAA length are presented. In addition, offline review of the exams and $3 \mathrm{D}$ visualization of the AAA are proposed in a comparable way with the CT workflow. Finally, in order to deal with limited field of view issues, multiple volume registrations are presented.

\section{METHOD}

\section{A. Material}

3D ultrasound aneurysm volumes are acquired using an IU22 system (Philips Ultrasound, Bothell, WA), and a 3D convex mechanical abdominal probe (V6-2). This probe is composed of a linear array of transducer elements that produce 2D slices. The mechanical angular scanning consists in a regular tilting of the transducer array in order to scan the volume. The slices are deflected by the same angle (tilt). The height of the acquired region is limited by the tilt amplitude of the transducers, controlled by the motor [6]. Standard field of view is about $10 \mathrm{~cm}$ in all directions.

Probe incidence influences image quality, as resolution of images is higher in 2D slices than in angular scanning direction. For comparison with standard 2D imaging, acquisitions were also made with a $2 \mathrm{D}$ convex abdominal probe (C5-2).

\section{B. Acquisitions}

30 patients, with 31 abdominal aortic or iliac aneurysms (13 AAA, 15 AAA after stenting, and 3 iliac aneurysms) were included in the study. For each aneurysm, 4 acquisitions were performed. Aneurysm was imaged in $2 \mathrm{D}$ in axial and longitudinal orientations. Then, 3D acquisitions were performed using 2 incidences of the mechanical probe: axial (fig. 1a) and longitudinal scanning (fig. 1c) with respect to the vessel orientation.

\section{Semi-automatic segmentation}

In order to extract quantified information from the volume acquisitions, it is necessary to segment the aneurysmal structure. Since manual segmentation of an aneurysm in 3D is a lengthy and tedious procedure, an interactive algorithm introduced in [7] was specifically designed to allow rapid 3D segmentations, optimally combining user interactions and image information. User interactions are as simple as clicks on the image, specifying whether a point in the image lies 


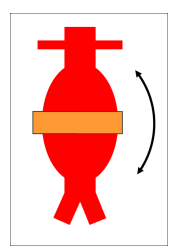

(a) 3D Axial

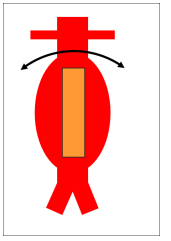

(c) 3D Longitudinal

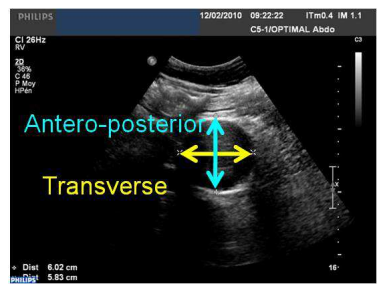

(b) $2 \mathrm{D}$ axial

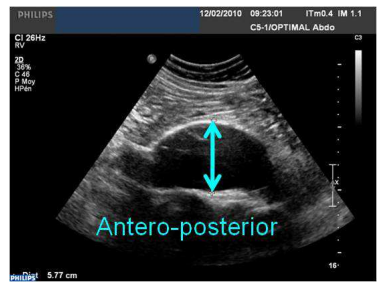

(d) 2D longitudinal
Fig. 1. 3D probe orientations and 2D reference acquisitions

inside or outside the aneurysm. In practice few interactions (less than 5 points) are needed to obtain satisfactory segmentations without tedious delineations. If needed, a few additional clicks either to locally adjust to contours or to correct for some "leakages" (commonly due to missing edge information) produce refined segmentation.

The segmentation method is based on an implicit representation of the aneurysm region as a linear combination of image dependent kernels (geodesic radial basis functions). The algorithm sequentially builds this representation as the user introduces points in the image. At the introduction of the $n^{\text {th }}$ point $x_{n}$, the algorithm computes an apodized geodesic distance $x \rightarrow \varphi\left(\left\|x-x_{n}\right\|_{g_{n}}\right)$ where $g_{n}$ is a metric function and $\varphi$ is a decreasing non-negative kernel. The aneurysm segmentation is then obtained by the interior $(\{x, \Phi(x) \geq 0\})$ of a linear combination of the previous interactions and the $n^{\text {th }}$ apodized geodesic distance:

$$
\Phi_{\lambda_{i=1 . . n}}(x)=\sum_{i=1}^{n} \lambda_{i} \varphi\left(\left\|x-x_{i}\right\|_{g_{i}}\right)
$$

The $n$ coefficients $\left\{\lambda_{i}\right\}_{i=1 . . n}$ are obtained by solving the constrained optimization problem:

$$
\min _{\lambda_{1}, \ldots, \lambda_{n}} E\left(\Phi_{\lambda_{i}}\right)=\int_{\Omega} H\left(\Phi_{\lambda_{i}}\right) r_{a}(I)+\int_{\Omega}\left(1-H\left(\Phi_{\lambda_{i}}\right)\right) r_{b}(I)
$$

where $I$ is the image intensity, $H$ is the Heaviside function, $r_{a}(I)=-\log P_{\Omega_{a}}(I), r_{b}(I)=-\log P_{\Omega \backslash \Omega_{a}}(I)$ are region terms, and $P_{\Omega_{a}}, P_{\Omega \backslash \Omega_{a}}$ are probability distributions of pixels intensity within the aneurysm and the exterior, respectively. This problem is minimized under a set of linear constraints specified as the user imposes a control point to be inside $\left(\Phi\left(x_{n}\right) \geq 0\right)$ or outside the aneurysm $\left(\Phi\left(x_{n}\right)<0\right)$.

Visual feedback of segmentation is real-time in 3D. The output of the semi-automatic segmentation is a surface $S$ representing the walls of the aneurysm (fig. 2).

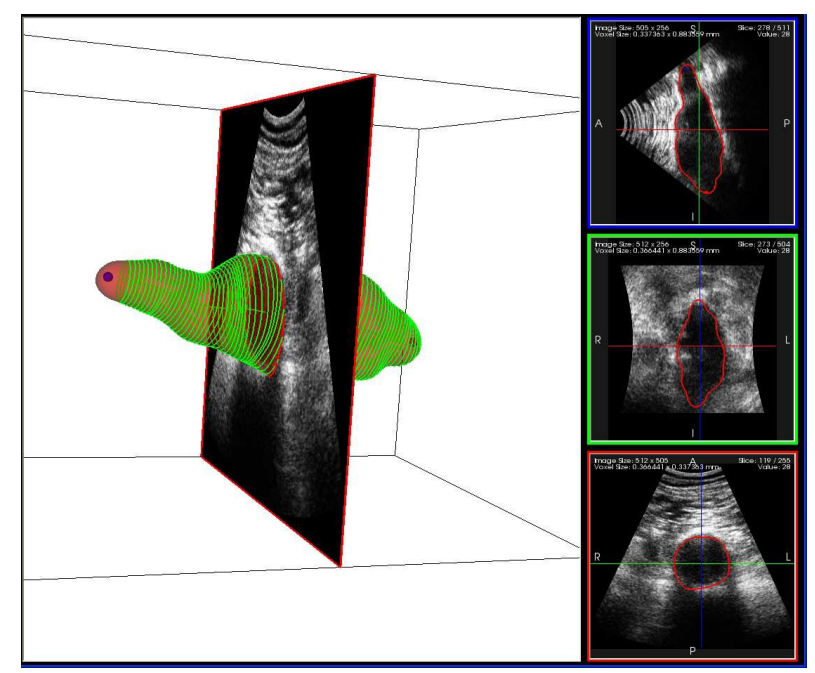

Fig. 2. 3D AAA visualization, centerline and cross-sections (green)

\section{Cross-sections extraction}

It has been shown in [8] that for 2D ultrasound acquisitions, the operator usually optimizes the acquisition incidence in order to provide an image showing the maximal size of the aneurysm. However, such a manual estimation of the best imaging plane does not ensure that the optimal incidence for measurement is selected. The principle of our approach is to provide an automatic selection of the optimal plane, avoiding to underestimate the aneurysm size.

After 3D aneurysm segmentation, the user is asked to click on proximal and distal extremities of the aneurysm. Clicks are used to generate a centerline of the surface $S$. The centerline is used as a referential for further quantification. We note $l$ the length of the aneurysm centerline and $s$ the curvilinear abscissa of a point on this centerline. By convention, $s=0$ at the proximal extremity of the aneurysm and $s=l$ at its distal extremity. For each $s$, a cross-section normal to the centerline is extracted, and the intersection of $S$ with the cross-section provides a 2D contour $C_{s}$ of the aneurysm. Quantification of the aneurysm from $3 \mathrm{D}$ volumes is based on the analysis of the set of contours $\left\{C_{s}\right\}$.

\section{E. Parameters definitions}

Selection of appropriate quantification parameters is essential to improve the accuracy of aneurysm assessement. In addition to providing a method to extract automatically the plane providing the largest aneurysm size, we propose to clarify the notions of measurement referential and of "diameter".

In $2 \mathrm{D}$, there is often an ambiguity between maximal diameter and diameters measured with anatomical referentials (such as antero-posterior and lateral). In our 3D approach, quantifications are made with respect to the centerline referential. Maximum diameter is the usual descriptor of aneurysm. However, this is based on the assumption that the cross-section of the aneurysm is circular. In many patients, the aneurysm can present an irregular geometry making the definition of 
the maximum diameter less relevant. We introduce the use of an elliptic model to characterize more accurately non circular cross-sections.

From each contour $C_{s}$, we extract 3 parameters :

- Real maximum diameter corresponding to the maximum distance between any couple of points on a contour.

- Short and long axes lengths obtained from the fitting of an elliptic model onto the contour, based on the method described in [9].

Each parameter being obtained for each cross-section, the output results in three functions $f_{\text {real }}(s), f_{\text {major }}(s)$ and $f_{\text {minor }}(s)$ (fig. 3).

For each function, the real maximum diameter, major and minor diameters are defined by $D X_{\text {real }}, D X_{\text {major }}$ and $D X_{\text {minor }}$ (eq. (3), (4), (5)), where A and L represent respectively an axial or a longitudinal acquisition.

$$
\begin{aligned}
& D X_{\text {real }}=\max _{s \in[0, l]}\left(f_{\text {real }}\right) \text { with } X \in\{A, L\} \\
& D X_{\text {major }}=\max _{s \in[0, l]}\left(f_{\text {major }}\right) \\
& D X_{\text {minor }}=f_{\text {minor }}\left(s=\operatorname{argmax}\left(f_{\text {major }}\right)\right)
\end{aligned}
$$

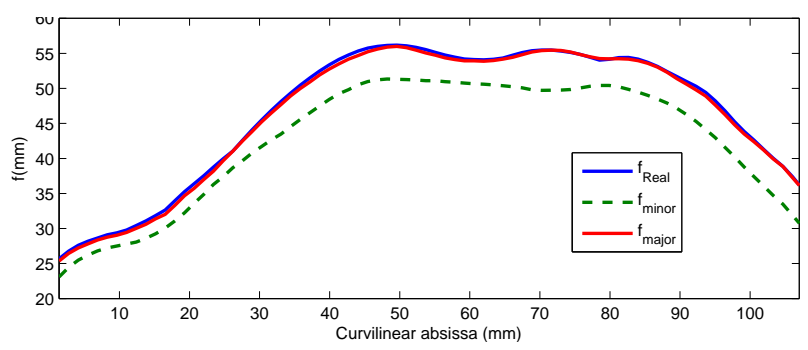

Fig. 3. Longitudinal quantification of diameter measurements

For comparison with 2D measurements, the following quantification is extracted from 2D acquisitions (fig. $1 \mathrm{~b}$ and $1 \mathrm{~d}$ ):

- in axial acquisition, maximal antero-posterior $d A_{\mathrm{AP}}$ and transverse diameters $d A_{\mathrm{T}}$

- in longitudinal acquisitions, maximal antero-posterior diameter $d L_{\mathrm{AP}}$

The 2D minimum and maximum diameters used for quantification are defined in eq. (6) and (7):

$$
\begin{aligned}
d_{\max } & =\max \left(d A_{\mathrm{AP}}, d A_{\mathrm{T}}, d L_{\mathrm{AP}}\right) \\
d_{\min } & =\min \left(d A_{\mathrm{AP}}, d A_{\mathrm{T}}, d L_{\mathrm{AP}}\right)
\end{aligned}
$$

\section{RESULTS}

\section{A. 3D Visualization and CT-like reconstructions}

3D ultrasound acquisitions provide a 3D visualization of the aneurysm geometry in space. Such a feature was previously limited to CT or MRI acquisitions (fig. 2). In addition, it is also possible to provide multi-planar reconstructions of the cross-sections, as is provided in CT workstations (fig. 4) for better assessement of the aneurysm geometry, making offline review possible.

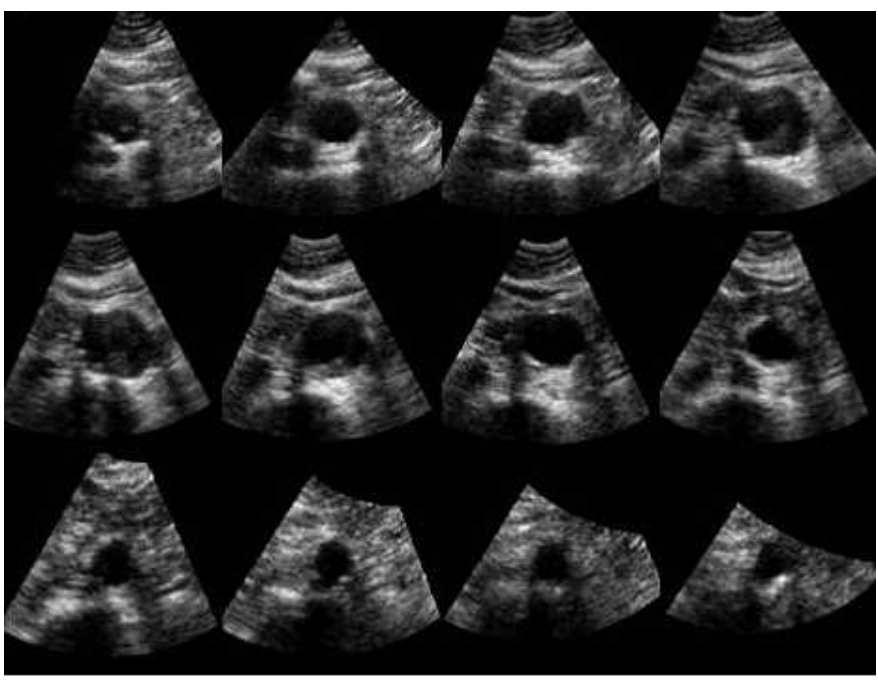

Fig. 4. Multi-planar reconstructions for assessement of AAA in a CT-like viewing mode

TABLE I

PEARSON COEFFICIENTS FOR 2D/3D CORRELATIONS

\begin{tabular}{|c||c|c|c|c|}
\hline & $D A_{\text {real }}$ & $D A_{\text {major }}$ & $D L_{\text {real }}$ & $D L_{\text {major }}$ \\
\hline$d_{\max }$ & 0.97471 & 0.97612 & 0.98050 & 0.98229 \\
\hline \hline & - & $D A_{\text {minor }}$ & - & $D L_{\text {minor }}$ \\
\hline$d_{\min }$ & - & 0.97788 & - & 0.97254 \\
\hline
\end{tabular}

\section{B. Quantification validation}

A first step towards the validation of the 3D quantification is to compare results with the conventional $2 \mathrm{D}$ measurements. For each patient, the 8 parameters $\left(D A_{\text {real }}, D A_{\text {major }}\right.$, $D A_{\text {minor }}, D L_{\text {real }}, D L_{\text {major }}, D L_{\text {minor }}, d_{\text {max }}$ and $\left.d_{\text {min }}\right)$ were computed. Correlations between 2D and 3D parameters are plotted in figure III-B. Minimum diameter in 2D is compared with the minor axis of the fitted ellipse. Maximum diameter in $2 \mathrm{D}$ is compared with the real diameter and the major axis of fitted ellipse. In order to assess the correlations, statistical analysis of the results was performed and Pearson correlation coefficients were calculated (table I). All statistical tests were significant $(p<0.0001)$ with very strong correlation coefficients $(>0.95)$.

\section{LIMITS}

Presented approach has intrinsic limits due to both ultrasound image quality and AAA physical size and geometry.

\section{A. Image quality limitations}

Quality of 3D ultrasound imaging of the aneurysm can be limited by presence of bowel gaz, depth, or complex geometries. Ultrasound beams may be tangent to the structures of interest. Regarding the segmentation, aneurysm walls identification can be difficult in presence of calcifications, or for obese patients. 

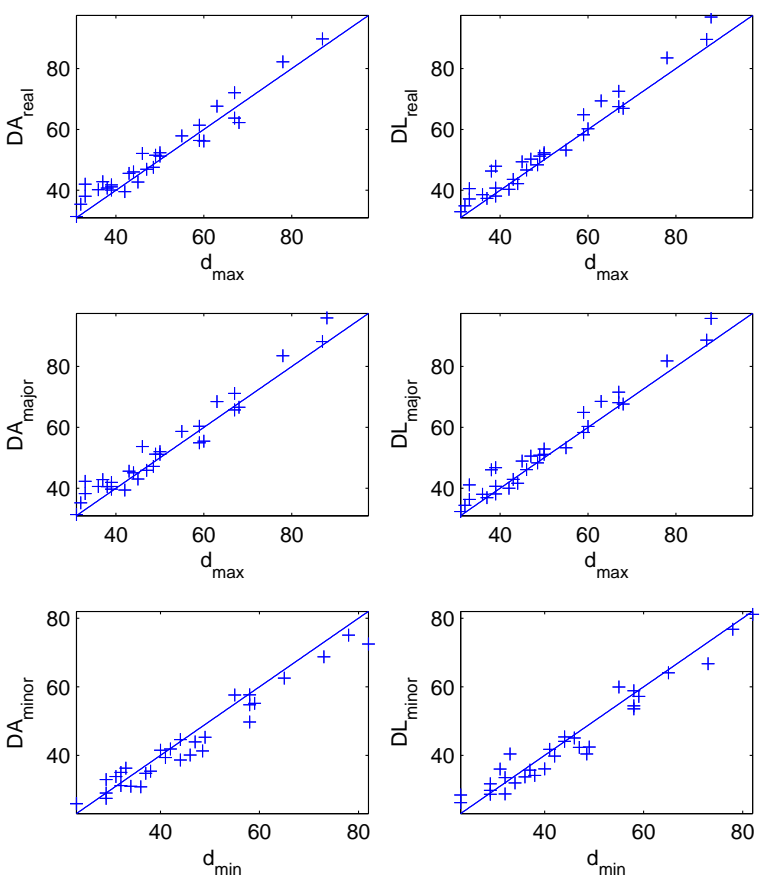

Fig. 5. Parameters correlations between 2D and 3D acquisitions, compared with $y=x$ function

\section{B. Limited field of view}

Depending on the geometry of the aneurysm with respect to the patient, the field of view can be too limited. In particular, very long or large aneurysms, or close to patient skin might not fit into a single volume. Two patients of the cohort present an aneurysm so large that it would not fit within the field of view of the probe. To overcome this difficulty, we propose to acquire multiple volumes in order to cover the full geometry of the aneurysm and then stitch together those volumes so that the whole geometry of the aneurysm is recovered.

In an attempt to validate this scheme, we performed an offline registration. A rigid $3 \mathrm{D}$ registration relying on the minimization of a simple distance (SSD) and taking data anisotropy into account was used to estimate the translation and rotation of the probe displacement between different views. Registrations were made between consecutive pair of images, and composed with respect to the central view. A panoramic volume was obtained, combining motion compensation, and data fusion. Visual inspection of final volume (fig. 6) showed good continuity of the data in all dimensions, despite the presence in some views of shadows and/or specular reflectors.

\section{CONCLUSiON}

3D ultrasound imaging of the AAA or iliac aneurysms and associated dedicated quantification has the potential to overcome some limitations of 2D imaging. In particular, automatic definition of the optimal imaging plane for measurement of the AAA size is a major input. Characterization of the aneurysm

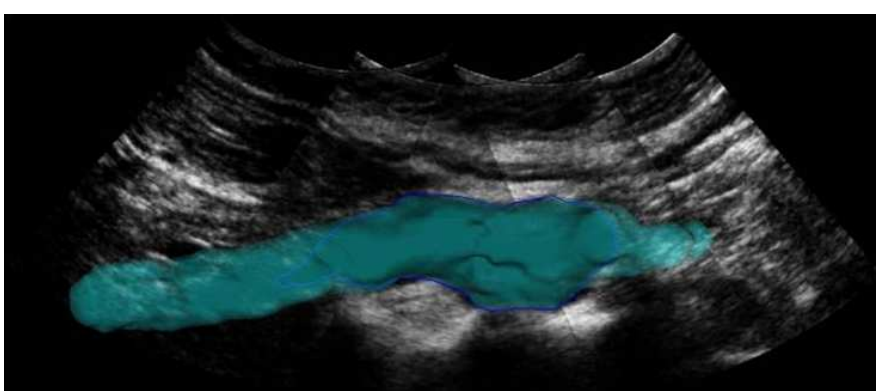

Fig. 6. Result of 3 volumes registration for a AAA too long to fit into a single acquisition

geometry using more advanced and precise descriptors such as elliptic model fitting was proposed to refine the geometric assessement of the aneuryms. Providing a full $3 \mathrm{D}$ visualization of the aneurysm geometry is also a major improvement for practicians, since offline review and measurements were previously reserved to $\mathrm{CT}$ acquisitions. The present evaluation has shown that 2D and 3D measurements are comparable, however, further validation is necessary in order to assess the improvement provided by $3 \mathrm{D}$ quantification in longitudinal follow-up of patients. More precisely, evaluating the variability of $3 \mathrm{D}$ measurements versus the $2 \mathrm{D}$ variability remains to be done.

\section{REFERENCES}

[1] J. Lindholt and P. Norman, "Screening for abdominal aortic aneurysm reduces overall mortality in men. a meta-analysis of the mid- and longterm effects of screening for abdominal aortic aneurysms." Eur J Vasc Endovasc Surg, 2008.

[2] M. Ellis, J. Powell, and R. Greenhalgh, "Limitations of ultrasonography in surveillance of small abdominal aortic aneurysms." Br J Surg, vol. 78, no. 5, pp. 614-616, 1991.

[3] F. Lederle, S. Wilson, G. Johnson, D. Reinke, F. Littooy, C. Acher, D. Ballard, L. Messina, I. Gordon, E. Chute, W. Krupski, S. Busuttil, G. Barone, S. Sparks, L. Graham, J. Rapp, M. Makaroun, G. Moneta, R. Cambria, R. Makhoul, D. Eton, H. Ansel, J. Freischlag, and D. Bandyk, "Immediate repair compared with surveillance of small abdominal aortic aneurysms." N Engl J Med, vol. 346, no. 19, pp. 1437-1444, 2002.

[4] The UK Small Aneurysm Trial Participants, "The u.k. small aneurysm trial: design, methods and progress. the uk small aneurysm trial participants." Eur J Vasc Endovasc Surg, vol. 9, no. 1, pp. 42-48, 1995.

[5] L. Sprouse 2nd, G. Meier 3rd, F. Parent, R. DeMasi, M. Glickman, and G. Barber, "Is ultrasound more accurate than axial computed tomography for determination of maximal abdominal aortic aneurysm diameter?" Eur J Vasc Endovasc Surg, vol. 28, no. 1, pp. 28-35, 2004.

[6] A. Long, L. Rouet, L. Gorin, E. Allaire, J. Becquemin, and P. Arbeille, "3D duplex scan for peripheral vascular applications: major progress or gimmick?" in Controversies and updates in vascular surgery 2010. Edizioni Minerva Medica, 01 2010, pp. 14-21.

[7] B. Mory, R. Ardon, A. Yezzi, and J. Thiran, "Non-euclidean imageadaptive radial basis functions for $3 \mathrm{D}$ interactive segmentation," in $I C C V$, 2009, pp. 787-794.

[8] S. Han, K. Patel, V. Rowe, S. Perese, A. Bond, and F. Weaver, "Ultrasound-determined diameter measurements are more accurate than axial computed tomography after endovascular aortic aneurysm repair." $J$ Vasc Surg, vol. 51, no. 6, pp. 1381-1389, 2010.

[9] R. Halî̃ and J. Flusser, "Numerically stable direct least squares fitting of ellipses," in Proc. 6th International Conference in Central Europe on Computer Graphics and Visualization. WSCG '98. Plzeň, Czech Republic: CZ, Feb. 1998, pp. 125-132. 